\title{
Some applications of bifurcation theory to ordinary differential equations of the fourth order
}

\author{
by Jolanta Przybycin (Kraków)
}

\begin{abstract}
The purpose of this paper is to study nonlinear eigenvalue problems for some fourth order ordinary diflerential equations. The general idea is to convert these equations into equivalent integral ones, for which we apply the results of Rabinowitz ([2], [3]). The main theorem connected with bifurcation from the line of trivial solutions is proved in Section 1. We show the existence of two families of continua of solutions, $\mathscr{C}_{k}^{+}$and $\mathscr{C}_{k}^{-}$, corresponding to the usual nodal properties and bifurcating from $\left(\mu_{k}, 0\right)$ where $\mu_{k}$ is the $k$-th eigenvalue of the linearization about 0 of the considered problem. In Section 2 we prove a related theorem for bifurcation from inlinity.
\end{abstract}

Introduction. Let $(E,\|\|)$ be a real Banach space and let $L: E \rightarrow E$ be a compact linear operator. If there exist $\mu \in \boldsymbol{R}$ and $0 \neq v \in E$ such that $v=\mu L v$, $\mu$ is said to be a real characteristic value of $L$. The set of real characteristic values of $L$ is denoted by $r(L)$. By the multiplicity of $\mu \in r(L)$ we understand the "algebraic multiplicity", i.e., $k_{\mu}=\operatorname{dim} \bigcup_{i=1}^{\infty} \operatorname{ker}(I-\mu L)^{i}$. The compactness of $L$ guarantees that $r(L)$ is discrete and each characteristic value $\mu \in r(L)$ is of finite multiplicity.

Consider the equation

$$
u=\lambda L u+H(\lambda, u),
$$

where $H: R \times E \rightarrow E$ is a compact operator satisfying the following condition:

$$
H(\lambda, u)=o(\|u\|) \text { at } u=0 \text { uniformly on bounded } \lambda \text { intervals. }
$$

By a solution of (0.1) is meant a pair $(\lambda, u) \in R \times E$ satisfying (0.1). In particular, $(0.1)$ has the line of trivial solutions $\mathscr{R}=\{(\lambda, 0) \mid \lambda \in \boldsymbol{R}\}$. If $(0.1)$ has a nontrivial solution in every neighbourhood of $(\lambda, 0)$, then $(\lambda, 0)$ is said to be a bifurcation point for $(0.1)$ with respect to the curve $\mathscr{R}$. It is well known that the possible bifurcation points for $(0.1)$ with respect to $\mathscr{R}$ lie on $\{(\mu, 0) \mid \mu \in r(L)\}$.

Let $\mathscr{S}$ denote the closure of the set of nontrivial solutions of $(0.1)$. A component of $\mathscr{S}$ is a maximal closed connected subset.

It was shown in [2] that:

THEOREM 0.3 (Rabinowitz). If $\mu \in r(L)$ is of odd multiplicity, $\mathscr{S}$ contains a component $\mathscr{C}_{\mu}$ which contains $(\mu, 0)$ and either 
(i) $\mathscr{C}_{\mu}$ is unbounded in $\boldsymbol{R} \times E$, or

(ii) $\mathscr{C}_{\mu}$ contains $(\hat{\mu}, 0)$ where $\mu \neq \hat{\mu} \in r(L)$.

Rabinowitz proved also a stronger result for a simple characteristic value $\mu$. To describe it precisely, let $v$ denote the normalized characteristic vector of $L$ corresponding to $\mu \in r(L), k_{\mu}=1$.

Let $E^{\prime}$ denote the dual space of $E,\langle$,$\rangle the duality between E^{\prime}$ and $E$, and $l \in E^{\prime}$ a normalized characteristic vector of $L^{*}$ corresponding to $\mu$. If $\hat{E}:=\{w \in E \mid$ $\langle l, w\rangle=0\}$, then $E=\{\operatorname{span} v\} \oplus \hat{E}$ and each $u \in E$ can be written as $u=\alpha v+w$, where $\alpha=\langle l, u\rangle$ and $w=u-\alpha v \in \hat{E}$.

THEOREM 0.4 (Rabinowitz). If $\mu \in r(L)$ is simple, $\mathscr{C}_{\mu}$ can be decomposed into two subcontinua $\mathscr{C}_{\mu}^{+}, \mathscr{C}_{\mu}^{-}$such that for some neighbourhood $\mathcal{O}$ of $(\mu, 0)$, the following implication is true:

$$
(\mu, 0) \neq(\lambda, u) \in \mathscr{C}_{\mu}^{+}\left(\mathscr{C}_{\mu}^{-}\right) \cap \mathcal{O} \Rightarrow(\lambda, u)=(\lambda, \alpha v+w),
$$

where $\alpha>0(\alpha<0)$ and $|\lambda-\mu|=o(1),\|w\|=o(|\alpha|)$ at $\alpha=0$.

Rabinowitz used the above results in his later work ([3]) to formulate theorems about bifurcation from infinity. Consider the equation

$$
u=\lambda L u+K(\lambda, u) \text {, }
$$

where $K: R \times E \rightarrow E$ is a continuous operator satisfying the condition

(0.6) $K(\lambda, u)=o(\|u\|)$ at $u=\infty$ uniformly on bounded $\lambda$ intervals.

We say $(\mu, \infty)$ is a bifurcation point for $(0.5)$ if every neighbourhood of $(\mu, \infty)$ contains solutions of $(0.5)$, i.e., there exists a sequence $\left(\lambda_{n}, u_{n}\right)$ of solutions of $(0.5)$ such that $\lambda_{n} \rightarrow \mu$ and $\left\|u_{n}\right\| \rightarrow \infty$. Let $\mathscr{T}$ denote the set of solutions of (0.5). The transformation $u \rightarrow u /\|u\|^{2}$ allows us to convert (0.5) into an equivalent equation of the form (0.1). This observation is the main tool in proofs of theorems about bifurcation from infinity.

THEOREM 0.7 (Rabinowitz). If $\mu \in r(L)$ is of odd multiplicity and $(\lambda, u) \rightarrow\|u\|^{2} K\left(\lambda, u /\|u\|^{2}\right)$ is compact, $\mathscr{T}$ has an unbounded component $\mathscr{D}_{\mu}$ which contains $(\mu, \infty)$. Moreover, if $\Lambda \subset \boldsymbol{R}$ is an interval such that $\Lambda \cap r(L)=\{\mu\}$ and $\mathscr{M}$ is a neighbourhood of $(\mu, \infty)$ whose projection on $\boldsymbol{R}$ lies in $\Lambda$ and whose projection on $E$ is bounded away from 0 , then either

(i) $\mathscr{D}_{\mu} \backslash \mathscr{M}$ is bounded in $\boldsymbol{R} \times E$ in which case $\mathscr{D}_{\mu} \backslash \mathscr{M}$ meets $\mathscr{R}$, or

(ii) $)_{\infty} \mathscr{D}_{\mu} \backslash \mathscr{M}$ is unbounded. If additionally $\mathscr{D}_{\mu} \backslash \mathscr{M}$ has a bounded projection on $\boldsymbol{R}$, then $\mathscr{D}_{\mu} \backslash \mathscr{M}$ contains $(\hat{\mu}, \infty)$, where $\mu \neq \hat{\mu} \in r(L)$.

THEOREM 0.8 (Rabinowitz). If $\mu \in r(L)$ is simple, $\mathscr{D}_{\mu}$ can be decomposed into two subcontinua $\mathscr{D}_{\mu}^{+}, \mathscr{D}_{\mu}^{-}$and there exists a neighbourhood $\mathcal{O} \subset \mathscr{M}$ such that the following implication is true:

$$
(\mu, \infty) \neq(\lambda, u) \in \mathscr{D}_{\mu}^{+}\left(\mathscr{D}_{\mu}^{-}\right) \cap \mathcal{O} \Rightarrow(\lambda, u)=(\lambda, \alpha v+w),
$$

where $\alpha>0(\alpha<0)$ and $|\lambda-\mu|=o(1),\|w\|=o(|\alpha|)$ at $|\alpha|=\infty$. 
1. Global bifurcation for a class of nonlinear eigenvalue problems for fourth order ordinary differential equations. Let $\mathscr{L}$ be a differential operator of the form $\mathscr{L}=\mathscr{L}_{1} \circ \mathscr{L}_{0}$, where $\mathscr{L}_{i}$ denotes the Sturm-Liouville operator defined by $\mathscr{L}_{i} u=-\left(p_{i} u^{\prime}\right)^{\prime}+q_{i} u, \quad i=0,1$. As usual we assume $p_{i} \in C^{3-2 i}[0, \pi]$, $q_{i} \in C^{2-2 i}[0, \pi]$ and $p_{i}>0, q_{i} \geqslant 0$ on $[0, \pi]$. We denote by (B.C.) either the boundary conditions

$$
u(0)=u(\pi)=u^{\prime}(0)=u^{\prime}(\pi)=0
$$

or the boundary conditions

$$
u(0)=u(\pi)=\mathscr{L}_{0} u(0)=\mathscr{L}_{0} u(\pi)=0 .
$$

Let $a$ be a positive continuous function on $[0, \pi]$. We assume additionally that the operator $\mathscr{L}$ is symmetric and positive definite (this is so in particular when $\left.\mathscr{L}_{0}=\mathscr{L}_{1}\right)$. Then the linear problem $\mathscr{L} v=\mu a v$ in $(0, \pi)$ together with the boundary conditions (B.C.) has an increasing sequence of simple eigenvalues $0<\mu_{1}<\mu_{2}<\ldots, \lim _{k \rightarrow \infty} \mu_{k}=\infty$. Any eigenfunction $v_{k}$ corresponding to $\mu_{k}$ has exactly $k-1$ simple zeros (nodes) in $(0, \pi)$.

The above result which is an extension of a classical theorem for the linear Sturm-Liouville problem was precisely formulated and proved in [1] and [4].

Now we consider the equation

$$
\mathscr{L} u=\lambda\left(a+h\left(\cdot, u, u^{\prime}, u^{\prime \prime}, u^{\prime \prime \prime}\right)\right) u \quad \text { in }(0, \pi)
$$

together with the boundary conditions (B.C.).

Assume that

(1.2) $h$ is a nonnegative continuous function on $[0, \pi] \times R^{4}$ and $\forall x \in[0, \pi]$, $h(x, 0,0,0,0)=0$.

By a solution of (1.1) we understand a pair $(\lambda, u) \in R \times\left(C^{4}[0, \pi] \cap(B . C).\right)$ satisfying (1.1).

Let $E=C^{3}[0, \pi] \cap(B . C) .$.$E is a Banach space equipped with its usual$ norm, $\|u\|_{3}:=\|u\|_{0}+\left\|u^{\prime}\right\|_{0}+\left\|u^{\prime \prime}\right\|_{0}+\left\|u^{\prime \prime \prime}\right\|_{0}$, where $\|u\|_{0}=\sup _{x \in[0, x]}|u(x)|$. Now we define a family of subsets of $E$ which are necessary for later considerations. Let

$S_{k}^{+}:=\{u \in E \mid u$ has exactly $k-1$ simple zeros in $(0, \pi), u$ is positive in a deleted neighbourhood of 0 and $\forall x \in[0, \pi], u^{2}(x)+\left[u^{\prime}(x)\right]^{2}+\left[u^{\prime \prime}(x)\right]^{2}$ $\left.+\left[u^{\prime \prime \prime}(x)\right]^{2} \neq 0\right\}$.

Then the sets $S_{k}^{+}, S_{k}^{-}:=-S_{k}^{+}$and $S_{k}=S_{k}^{+} \cup S_{k}^{-}$are open in $E$ and $S_{k} \cap S_{j}=\varnothing$ for $k \neq j$. Any eigenfunction $v_{k}$ corresponding to $\mu_{k}$ belongs to $S_{k}$. By requiring $v_{k} \in S_{k}^{+}$and $\left\|v_{k}\right\|_{3}=1, v_{k}$ is uniquely determined.

Notice that it is sufficient to search for solutions of (1.1) in $R \times E$.

Namely, by using the Green function $g$ of $\mathscr{L}$ together with the boundary conditions (B.C.) equation (1.1) can be converted into an equivalent integral 
equation in $\boldsymbol{R} \times E$ :

$$
u(\cdot)=\lambda \int_{0}^{\pi} g(\cdot, y)\left[a(y)+h_{u}(y)\right] u(y) d y \equiv \lambda L u+H(\lambda, u),
$$

where

$$
\begin{aligned}
L u & =\int_{0}^{\pi} g(\cdot, y) a(y) u(y) d y \\
H(\lambda, u) & =\lambda \int_{0}^{\pi} g(\cdot, y) h_{u}(y) u(y) d y \\
h_{u}(y) & =h\left(y, u(y), u^{\prime}(y), u^{\prime \prime}(y), u^{\prime \prime \prime}(y)\right) .
\end{aligned}
$$

The above symbols for the operators in (1.3) are introduced consciously. Namely we show that equation (1.3) is of the form (0.1). It is easy to prove, applying the Arzelà-Ascoli theorem, that $L: E \rightarrow E$ is compact as is $H$ : $\boldsymbol{R} \times E \rightarrow E$.

We must show that $H$ satisfies (0.2). Let $A$ denote a bounded interval in $\boldsymbol{R}$ and fix $\varepsilon>0$. By (1.2) we have

$$
\exists \delta>0 \quad|\xi|+|\eta|+|\gamma|+|\zeta|<\delta \Rightarrow \mid h(x, \xi, \eta, \gamma, \zeta \xi \mid<\varepsilon(|\xi|+|\eta|+|\gamma|+|\zeta|) .
$$

Hence, for $(\lambda, u) \in \Lambda \times S=\left\{(\lambda, u) \in \Lambda \times E \mid\|u\|_{3}<\delta\right\}$ we obtain the estimate

$$
\begin{aligned}
\|H(\lambda, u)\|_{3} & =\sum_{i=0}^{3} \max _{x \in[0, \pi]}|\lambda|\left|\int_{0}^{\pi} \frac{\partial^{l} g}{\partial x^{i}}(x, y) h_{u}(y) u(y) d y\right| \\
& \leqslant C \int_{0}^{\pi}\left|h_{u}(y)\right||u(y)| d y \leqslant C \pi \varepsilon\|u\|_{3},
\end{aligned}
$$

where $C$ depends on $\Lambda$ and on bounds for $g, \partial g / \partial x, \partial^{2} g / \partial x^{2}, \partial^{3} g / \partial x^{3}$.

Therefore $H(\lambda, u)=o\left(\|u\|_{3}\right)$ at $u=0$ uniformly on bounded $\lambda$ intervals. Notice that the eigenvalues of $\mathscr{L}$ are equal to the characteristic values of $L$ and are simple. Hence all $\mu_{k} \in r(L)$ satisfy the hypotheses of Theorem 0.3 and accordingly there exists a component $\mathscr{C}_{k}$ of $\mathscr{S}$ which contains $\left(\mu_{k}, 0\right)$ and is either unbounded in $R \times E$ or contains $\left(\mu_{j}, 0\right)$, where $j \neq k$. Actually only the first alternative is possible as will be shown next. For this purpose we require two important lemmas.

LEMMA 1.4. There exists a neighbourhood $U_{k}$ of $\left(\mu_{k}, 0\right)$ such that the following implication is true:

$$
(\lambda, u) \in U_{k} \cap \mathscr{S} \text { and } u \neq \equiv \Rightarrow u \in S_{k} .
$$

Proof. If not, there exists a sequence $\left(\lambda_{n}, u_{n}\right) \in \mathscr{S}$ such that $0 \neq u_{n} \notin S_{k}$ and $\left(\lambda_{n}, u_{n}\right) \rightarrow\left(\mu_{k}, 0\right)$ in $R \times E$ as $n \rightarrow \infty$. Writing equation (1.3) for $\left(\lambda_{n}, u_{n}\right)$ and 
dividing it by $\left\|u_{n}\right\|_{3}$ yields

$$
\frac{u_{n}}{\left\|u_{n}\right\|_{3}}=\lambda_{n} L \frac{u_{n}}{\left\|u_{n}\right\|_{3}}+\frac{H\left(\lambda_{n}, u_{n}\right)}{\left\|u_{n}\right\|_{3}} .
$$

Since $L$ is compact and $H$ satisfies (0.2), the left-hand side of the above equation has a convergent subsequence $u_{n_{p}} /\left\|u_{n_{p}}\right\|_{3} \stackrel{E}{\rightarrow} w$ with $\|w\|_{3}=1$ and $w=\mu_{k} L w$. Consequently $w= \pm v_{k} \in S_{k}$. Since $S_{k}$ is open, $u_{n_{p}} \in S_{k}$ for $p$ sufficiently large, contrary to the assumption.

LEMMA 1.5. If $(\lambda, u) \in \boldsymbol{R} \times \partial S_{k}$ is a solution of $(1.1)$, then $u \equiv 0$.

Proof. Assume that $(\lambda, u) \in \boldsymbol{R} \times \partial S_{k}$ is a nontrivial solution of (1.1). Consider the linear eigenvalue problem $\mathscr{L} v=\mu\left(a+h\left(\cdot, u, u^{\prime}, u^{\prime \prime}, u^{\prime \prime \prime}\right)\right) v$ in $(0, \pi)$ with the boundary conditions (B.C.).

Since $(\mu, v)=(\lambda, u)$ is an eigenpair of the above problem, we have $u \in \bigcup_{k=1}^{\infty} S_{k}$, which is impossible.

Now we can prove the main global bifurcation theorem for (1.1).

THEOREM 1.6. For every $k \in N$ there exists an unbounded component of $\mathscr{S}$, $\mathscr{C}_{k}$, bifurcating from $\left(\mu_{k}, 0\right)$ and contained in $\left(R \times S_{k}\right) \cup\left\{\left(\mu_{k}, 0\right)\right\}$.

Proof. It suffices to show that $\mathscr{C}_{k} \subset\left(R \times S_{k}\right) \cup\left\{\left(\mu_{k}, 0\right)\right\}$. Suppose $\mathscr{C}_{k} \neq\left(\boldsymbol{R} \times S_{k}\right) \cup\left\{\left(\mu_{k}, 0\right)\right\}$. By Lemma 1.4, $\mathscr{C}_{k} \cap U_{k} \subset\left(R \times S_{k}\right) \cup\left\{\left(\mu_{k}, 0\right)\right\}$. Since $\mathscr{C}_{k}$ is connected, there must exist $\left(\mu_{k}, 0\right) \neq(\lambda, u) \in \mathscr{C}_{k} \cap\left(R \times \partial S_{k}\right)$ and $(\lambda, u)=\lim _{n \rightarrow \infty}\left(\lambda_{n}, u_{n}\right)$. By Lemma $1.5, u \equiv 0$. Hence $\lambda=\mu_{j}, j \neq k$. Applying Lemma 1.4 again we find that $\left(\lambda_{n}, u_{n}\right) \in U_{j} \cap\left(\boldsymbol{R} \times S_{j}\right)$ for $n$ large enough, which is impossible, and the proof is complete.

Remark. By using Theorem 0.4 we can decompose the component $\mathscr{C}_{k}$ into two unbounded subcontinua $\mathscr{C}_{k}^{+}, \mathscr{C}_{k}^{-}$such that $\mathscr{C}_{k}^{ \pm} \subset\left(R \times S_{k}^{ \pm}\right) \cup\left\{\left(\mu_{k}, 0\right)\right\}$.

EXAmple. Consider the equation $u^{(4)}=\lambda\left(1+u^{2}+u^{\prime 2}\right) u$ in $(0, \pi)$ with the boundary conditions $u(0)=u(\pi)=u^{\prime \prime}(0)=u^{\prime \prime}(\pi)=0$.

It is easy to see that $(1,0)$ is a bifurcation point of this problem. In every neighbourhood of $(1,0)$ there exist nontrivial solutions of the form $\left(1 /\left(1+\gamma^{2}\right), \gamma \sin x\right), \gamma \in \boldsymbol{R}$. Hence

$$
\begin{aligned}
& \mathscr{C}_{1}^{+}=\left\{\left(\frac{1}{1+\gamma^{2}}, \gamma \sin x\right) \mid \gamma \geqslant 0\right\} \subset\left(\boldsymbol{R} \times S_{1}^{+}\right) \cup\{(1,0)\}, \\
& \mathscr{C}_{1}^{-}=\left\{\left(\frac{1}{1+\gamma^{2}}, \gamma \sin x\right) \mid \gamma \leqslant 0\right\} \subset\left(\boldsymbol{R} \times S_{1}^{-}\right) \cup\{(1,0)\} .
\end{aligned}
$$

2. Bifurcation from infinity for nonlinear eigenvalue problems with differential operators of the fourth order. Consider the nonlinear equation

$$
\mathscr{L} u \equiv \mathscr{L}_{1} \circ \mathscr{L}_{0} u=\lambda a u+k\left(\cdot, u, u^{\prime}, u^{\prime \prime}, u^{\prime \prime \prime}, \lambda\right) \quad \text { in }(0, \pi)
$$

together with the boundary conditions (B.C.). 
All the assumptions on $\mathscr{L}$ and $a$ from the previous section are still valid. Assume additionally that the right-hand side of (2.1) is a continuous function on $[0, \pi] \times R^{5}$ and

$k(x, \xi, \eta, \gamma, \zeta, \lambda)=o(|\xi|+|\eta|+|\gamma|+|\zeta|)$ at $(\xi, \eta, \gamma, \zeta)=\infty$ uniformly in $x \in[0, \pi]$ and $\lambda \in \Lambda$ for any bounded interval $\Lambda \subset \boldsymbol{R}$.

Analogously to Section 1 we can convert (2.1) into an equivalent operator equation in $R \times E=R \times\left(C^{3}[0, \pi] \cap(B . C).\right)$;

$$
u(\cdot)=\int_{0}^{\pi} g(\cdot, y)\left[\lambda a(y) u(y)+k_{u}(y, \lambda)\right] d y \equiv \lambda L u+K(\lambda, u),
$$

where $k_{u}(y, \lambda)=k\left(y, u(y), u^{\prime}(y), u^{\prime \prime}(y), u^{\prime \prime \prime}(y), \lambda\right)$. Let $\mathscr{T}$ denote the set of solutions of (2.1) in $R \times E$.

THEOREM 2.4. For each integer $k \in N$ there exists a component $\mathscr{D}_{k}$ of $\mathscr{T}$ which contains $\left(\mu_{k}, \infty\right)$ and satisfies the conclusions of Theorems 0.7 and 0.8 . Moreover, there exists a neighbourhood of $\left(\mu_{k}, \infty\right)$, $\mathcal{O}$, such that $\mathscr{D}_{k}^{ \pm}$ $\cap \mathcal{O} \subset\left(R \times S_{k}^{ \pm}\right) \cup\{(\mu, \infty)\}$.

Proof. It is necessary to show that (2.3) is of the form (0.5) and the operator $\hat{K}:(\lambda, u) \rightarrow\|u\|_{3}^{2} K\left(\lambda, u /\|u\|_{3}^{2}\right)$ is compact. Clearly $K$ is continuous on $\boldsymbol{R} \times E, L$ is compact and linear. To show that $K$ satisfies (0.6), let $\Lambda \subset \boldsymbol{R}$ be a bounded interval and fix $\varepsilon>0$. (2.2) implies that

$$
\exists \delta>0 \quad|\xi|+|\eta|+|\gamma|+|\zeta|>\delta \Rightarrow|k(x, \xi, \eta, \gamma, \zeta, \lambda)|<\varepsilon(|\xi|+|\eta|+|\gamma|+|\zeta|) .
$$

Moreover, by the continuity of $k$

$$
|k(x, \xi, \eta, \gamma, \zeta, \lambda)| \leqslant M \quad \text { for }|\xi|+|\eta|+|\gamma|+|\zeta| \leqslant \delta .
$$

Choosing $\delta_{1}$ so large that $M / \delta_{1}<\varepsilon$ and setting $S:=\left\{u \in E \mid\|u\|_{3} \geqslant \delta_{1}\right\}$ we obtain for $(\lambda, u) \in \Lambda \times S$ the following estimate:

$$
\|K(\lambda, u)\|_{3}=\sum_{i=0}^{3} \max _{x \in[0, \pi]}\left|\int_{0}^{\pi} \frac{\partial^{i}}{\partial x^{i}} g(x, y) k_{u}(y, \lambda) d y\right|
$$

$\leqslant C \int_{|u(y)|+\left|u^{\prime}(y)\right|+\left|u^{\prime \prime}(y)\right|+\left|u^{\prime \prime \prime}(y)\right| \leqslant \delta}\left|k_{u}(y, \lambda)\right| d y+C \int_{|u(y)|+\left|u^{\prime}(y)\right|+\left|u^{\prime \prime}(y)\right|+\left|u^{\prime \prime \prime}(y)\right|>\delta}\left|k_{u}(y, \lambda)\right| d y$

$\leqslant C \pi\left(M+\varepsilon\|u\|_{3}\right)<2 C \pi \varepsilon\|u\|_{3}$,

where $C$ depends on bounds for $g, \partial g / \partial x, \partial^{2} g / \partial x^{2}, \partial^{3} g / \partial x^{3}$. Hence $K$ satisfies (0.6). To verify that $\hat{K}$ is compact, note that if $\Lambda \subset R$ is bounded, the image of $\left\{(\lambda, u) \in \Lambda \times E \mid \quad \varrho_{1} \leqslant\|u\|_{3} \leqslant \varrho_{2}\right\}$ under $\hat{K}$ is relatively compact for any $0<\varrho_{1} \leqslant \varrho_{2}<\infty$. Thus we need only prove that $\mathbb{K}\left(\Lambda \times \bar{B}_{1 / \delta_{1}}\right)$ is relatively compact in $E, \bar{B}_{1 / \delta_{1}}=\left\{u \in E \mid\|u\|_{3} \leqslant 1 / \delta_{1}\right\}$. From (2.5) we obtain

$$
\|\hat{K}(\lambda, u)\|_{3}=\|u\|_{3}^{2}\left\|K\left(\lambda, u /\|u\|_{3}^{2}\right)\right\|_{3}<2 C \pi \varepsilon / \delta_{1} \quad \text { for }(\lambda, u) \in \Lambda \times \bar{B}_{1 / \delta_{1}} .
$$


Hence $\hat{K}\left(\Lambda \times \bar{B}_{1 / \delta_{1}}\right)$ is bounded in $E$. Moreover, $z=\hat{K}(\lambda, u)$ satisfies

$$
\mathscr{L}_{z}=\|u\|_{3}^{2} k\left(\cdot, \frac{u}{\|u\|_{3}^{2}}, \frac{u^{\prime}}{\|u\|_{3}^{2}}, \frac{u^{\prime \prime}}{\|u\|_{3}^{2}}, \frac{u^{\prime \prime \prime}}{\|u\|_{3}^{2}}, \lambda\right) .
$$

Notice that $\left(\lambda, u /\|u\|_{3}^{2}\right) \in \Lambda \times S$. Hence, solving the above equation for $z^{(4)}$ we obtain uniform bounds for the fourth derivatives of $\hat{K}(\lambda, u)$ in $\Lambda \times \bar{B}_{1 / \delta_{1}}$. Applying the Arzelà-Ascoli theorem we deduce at once the compactness of $\hat{K}$.

So Theorems 0.7 and 0.8 are applicable here. It remains to show that for $\mathcal{O}$ possibly still smaller than in the conclusion of Theorem 0.8 the following implication is true:

$$
(\lambda, u) \in \mathcal{O} \cap \mathscr{D}_{k}^{+}\left(\mathscr{D}_{k}^{-}\right) \Rightarrow u \in S_{k}^{+}\left(S_{k}^{-}\right) .
$$

By Theorem 0.7 we know that

$(\lambda, u)=\left(\lambda, \alpha v_{k}+w\right), \quad$ where $\alpha>0(\alpha<0)$ and $\|w\|_{3}=o(|\alpha|)$ at $|\alpha|=\infty$.

Since $S_{k}^{+}\left(S_{k}^{-}\right)$is open, $v_{k}+w / \alpha \in S_{k}^{+}\left(S_{k}^{-}\right)$for $\alpha$ near $\infty$. Therefore $u=\alpha v_{k}+w \in S_{k}^{+}\left(S_{k}^{-}\right)$. Thus the proof is complete.

Remark. Unlike in Theorem 1.6, for bifurcation from infinity the inclusion $\mathscr{D}_{k}^{ \pm} \subset\left(R \times S_{k}^{ \pm}\right) \cup\left\{\left(\mu_{k}, \infty\right)\right\}$ need not hold. Look at the following example:

$$
u^{(4)}=\lambda(u+1) \quad \text { in }(0, \pi)
$$

with the boundary conditions $u(0)=u(\pi)=u^{\prime \prime}(0)=u^{\prime \prime}(\pi)=0$.

For $\lambda \neq k^{4}$ the solution is unique and given by

$$
u_{\lambda}(x)=-1+\cos \sqrt[4]{\lambda} x+\frac{1-\cos \sqrt[4]{\lambda} \pi}{\sin \sqrt[4]{\lambda} \pi} \sin \sqrt[4]{\lambda} x
$$

Hence

$$
\mathscr{D}_{1}^{+}=\left\{\left(\lambda, u_{\lambda}\right) \mid \lambda \in(0,1)\right\} \cup\{(0,0)\} \cup\{(1, \infty)\} \notin\left(\boldsymbol{R} \times S_{1}^{+}\right) \cup\{(1, \infty)\} .
$$

COROLlary. If we assume additionally that $k(x, \xi, \eta, \gamma, \zeta, \lambda)=$ $\lambda h(x, \xi, \eta, \gamma, \zeta) \xi$, where $h$ satisfies (1.2), then $\mathscr{D}_{k}^{ \pm} \backslash \mathcal{O}$ contains a subcontinuum lying in $R \times S_{k}^{ \pm}$which is unbounded or intersects the line of trivial solutions $\mathscr{R}$ at $\left(\mu_{k}, 0\right)$.

Proof. It is known that $\mathscr{D}_{k}^{ \pm} \cap \mathcal{O} \subset\left(R \times S_{k}^{ \pm}\right) \cup\left\{\left(\mu_{k}, \infty\right)\right\}$. Let $\mathscr{E}_{k}^{+}$denote the maximal subcontinuum of $\mathscr{D}_{k}^{+}$contained in $R \times S_{k}^{+}$. Suppose $\mathscr{E}_{k}^{+} \backslash \mathcal{O}$ is bounded. Then there exists $(\lambda, u) \in \partial \mathscr{E}_{k}^{+} \cap\left(R \times \partial S_{k}^{+}\right)$. By Lemma $1.5, u \equiv 0$. Hence $\left(\mathscr{E}_{k}^{+} \backslash \mathcal{O}\right) \cap \mathscr{R} \neq \varnothing$. Notice that only $\left(\mu_{k}, 0\right)$ can be the limit of solutions $(\lambda, u) \in \boldsymbol{R} \times S_{k}^{+}$. Hence $\left(\mathscr{E}_{k}^{+} \backslash \mathcal{O}\right) \cap \mathscr{R}=\left\{\left(\mu_{k}, 0\right)\right\}$.

EXAMPLE. Consider the equation

$$
u^{(4)}=\lambda\left(1+\frac{u^{2}}{\left(1+u^{2}\right)^{2}}\right) u \quad \text { in }(0, \pi)
$$


together with the boundary conditions $u(0)=u(\pi)=u^{\prime \prime}(0)=u^{\prime \prime}(\pi)=0$. Notice that $\mu_{k} / \lambda \in[1,2]$. Hence $\lambda \in\left[\frac{1}{2} \mu_{k}, \mu_{k}\right]$. Therefore $\mathscr{D}_{k}^{ \pm} \subset\left[\frac{1}{2} \mu_{k}, \mu_{k}\right] \times E$ and $\mathscr{D}_{k}^{ \pm}$meets $\mathscr{R}$ at $\left(\mu_{k}, 0\right)$.

\section{References}

[1] J. B oche nek, Nodes of eigenfunctions of a certain class of ordinary differential equations of the fourth order, Ann. Polon. Math. 29 (1975), 349-356.

[2] P. H. R abin owitz, Some aspects of nonlinear eigenvalue problems, Rocky Mountain J. Math. 3 (1973), 161-202.

[3] -, On bifurcation from infinity, J. Differential Equations 14 (1973), 462-475.

[4] T. Winiarska, Zero points of the eigenfunctions of a composition of operators of the Sturm-Liouville type, Zeszyty Naukowe Uniw. Jagiell. 19 (1977), 13-31.

INSTITUTE OF MATHEMATTCS

ACADEMY OF MINING AND METALLURGY

Mickieqicza 30, 30-059. Kraków, Poland

Reçu par la Rédaction le 06.06.1989 